\title{
Leukocytoclastic vasculitis after the third dose of CoronaVac vaccination
}

\author{
T. Oskay ${ }^{1}\left[\right.$ ] M. Isık ${ }^{2}$ (1)
}

Received: 21 October 2021 / Revised: 8 November 2021 / Accepted: 9 November 2021 / Published online: 1 December 2021

(c) International League of Associations for Rheumatology (ILAR) 2021

\section{Dear Editor:}

A 77-year-old male who developed eruptive skin lesions was admitted to our clinic. Two weeks after receiving the third injection of the vaccine (July 15), he complained of arthralgia, myalgia, and general fatigue and also experienced intestinal symptoms such as bloody diarrhea and abdominal pain. He reported a mildly itchy rash with his first (February 12) and second-dose (March 16) immunizations, without fever, difficulty breathing, edema, oliguria, or other systemic symptoms. He denied any prior history of vaccinations, food, drug, or environmental allergies, or systemic autoimmune diseases, nor any other recent changes to his medications.

Dermatologic examination revealed diffuse palpable, tender, non-blanching violaceous coalescent patches on the thighs, calves, feet, and hands. There were also bullous hemorrhagic lesions distributed bilaterally on the extensor sides of the lower legs and feet (Fig. 1A, B). The rest of the physical examination was unremarkable.

Pathologic laboratory testing revealed elevated C-reactive protein levels of $60 \mathrm{mg} / \mathrm{L}$ (normal: 6), D-dimer levels of 6028 (normal: 500), and sedimentation rates of $44 \mathrm{~mm} / \mathrm{h}$ (normal: 20). The white blood cell count, peripheral smear, liver, and renal function tests were within normal ranges. Rheumatologic tests showed positive antinuclear antibodies (ANA: 1/ 100) and PM-SCl++, while other autoimmune workup revealed no pathologic findings including complement $\mathrm{CH} 50, \mathrm{C} 3, \mathrm{C} 4$, rheumatic factor, antidsDNA,

$\triangle$ T. Oskay

tboskay@hotmail.com

M. Isık

Metin1721978@yahoo.com

1 Department of Dermatology, Bayındır Health Group, Ankara, Turkey

2 Department of Rheumatology, Yuzuncu Yil Hospital, Ankara, Turkey
c/p-ANCA, antiphospholipid antibodies, and ENA panel - e.g. Jo1, U1-RNP-, SCL70-, Sm-, and Ro/La-antibodies. Cryoglobulin and screening for viral infections (hepatitis $\mathrm{A}, \mathrm{B}$, and C, CMV, EBV, Coxsackie, parvovirus B19, and HIV) were also negative. A nasal swab proved negative for the SARS-CoV-2 virus. CT scans of the thorax and abdominopelvic yielded normal results. Dermatopathologic examination of the skin biopsy was compatible with small vessel LCV in the dermis showing plump endothelial cells surrounded by a perivascular mixed inflammatory infiltrate with karyorrhectic debris and extravasation of red blood cells (Fig. 1C, D). Direct immunofluorescence did not reveal any deposits in the vessels. His abdominal pain and stool tests on occult blood-positive favor occult bleeding associated with LCV of gastrointestinal involvement.

From the correlation of the clinical presentation, laboratory findings, and recent COVID-19 vaccine, we established the diagnosis of cutaneous and gastrointestinal LCV, possibly triggered by the third dose of immunization, by excluding possible triggers such as concomitant medication, other infectious, malignant, and autoimmune causes. As our patient presented with pronounced skin and gastrointestinal involvement, we treated him with oral prednisolone at a dose of $0.5 \mathrm{mg} / \mathrm{kg} / \mathrm{day}$, with a rapid response. At a follow-up 14 days later, all clinical manifestations and laboratory findings were resolved.

So far, cutaneous vasculitis presenting with typical skin lesions has been well reported in mild as well as fulminant COVID-19 infections. Vasculitis in COVID-19 has been attributed to SARS-CoV-2-associated endothelitis, which could be because of the virus directly invading the endothelium or owing to an inflammatory response that results in immune complex deposits in the vessels [1].

Recently, COVID-19 vaccinations associated with LCV and autoimmune adverse effects have been described, but they are relatively rare. The Coronavac vaccine (Sinovac, China) contains inactivated whole or split virion representatives of COVID-19 viruses, as well as several adjuvants 
Fig. 1 A Dermatological examination reveals multiple, discrete to confluent palpable purpuras distributed symmetrically over both lower limbs. B Bullous hemorrhagic lesions distributed bilaterally on the extensor sides of the lower legs and feet. C Histopathology of a lesion suggests leukocytoclastic vasculitis with small vessels in the dermis showing plump endothelial cells surrounded by a perivascular mixed inflammatory infiltrate with karyorrhectic debris and extravasation of RBCs. (H \& E, 200x). D Inflammatory cells comprising neutrophils and lymphocytes infiltrating the vessel wall. Leukocytoclasia is seen. (H \& E, 400x)

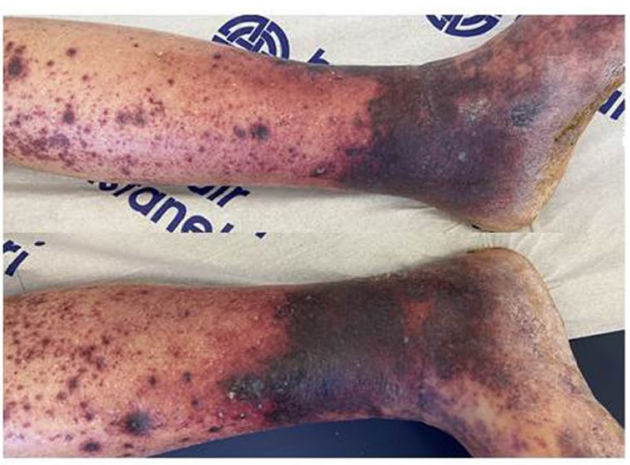

A

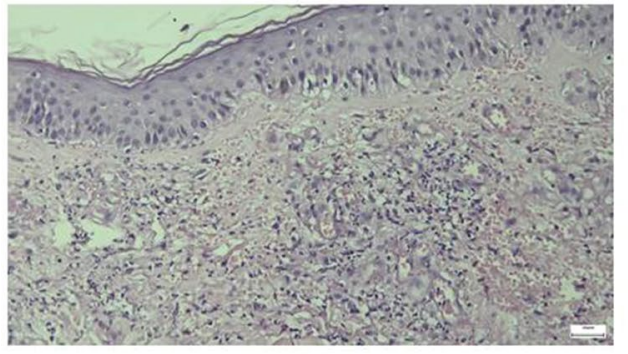

C

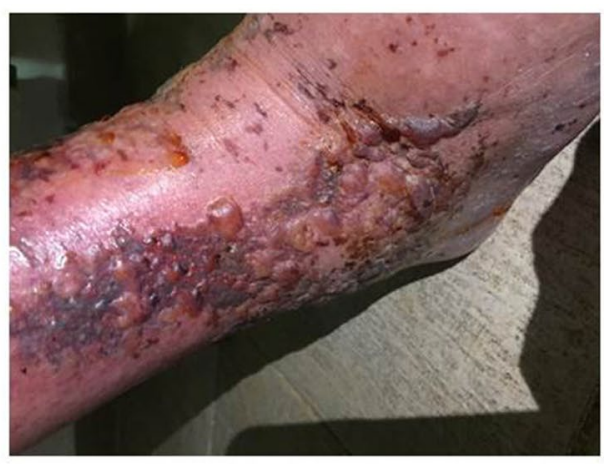

B

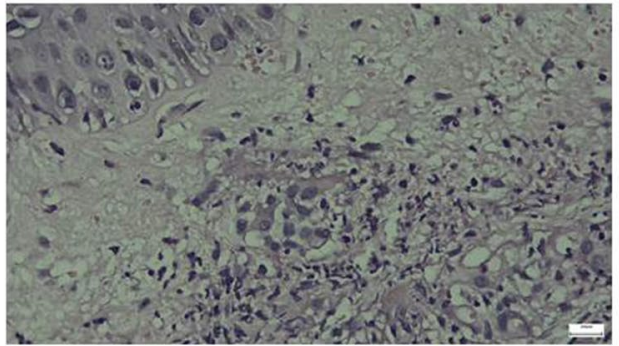

D (aluminum) that are unlikely to cause immunopathology. It's still unclear if it's a hypersensitivity reaction to the SARSCoV-2 spike protein or the other vaccination component [2]. The COVID-19 vaccine may provoke hyper-activation of the immune system secondary to cross-reactivity and molecular mimicry between the virus and self-antigens, consequently triggering autoimmune disorders such as vasculitis in genetically predisposed individuals [3].

To our knowledge, there were few previous case reports of vaccine-associated reactivation or new-onset $\mathrm{LCV}$ or IgA vasculitis after COVID-19 with mRNA or inactivated SARS-CoV-2 vaccines at the first or second doses, but none at the third dose [4-9]. In addition, in some case reports, mRNA COVID-19 vaccinations have been linked to newonset or recurrent ANCA-associated vasculitis with kidney impairment [10]. Compared to other reported cases, he developed GIS involvement and responded faster to corticosteroids. In our case, the large antigenic trigger because of the booster re-exposure of the third dose may have contributed to the development of cutaneous LCV with GIS involvement without known predispositions to the type 3 hypersensitivity.

The purpose of this case report is to increase awareness of the potential side effects of the vaccine. However, more studies are needed to better characterize the adverse effects of these vaccines on elderly individuals.

Author contribution The authors contributed equally to all aspects of the article.

\section{Declarations}

Ethics approval and consent to participate Written informed consent was taken from the patient to present this case report.

Disclosures None.

\section{References}

1. Drenovska K, Schmidt E, Vassileva S (2020) Covid-19 pandemic and the skin. Int J Dermatol 59:1312-1319

2. Niebel D, Novak N, Wilhelmi J et al (2021) Cutaneous adverse reactions to COVID-19 vaccines: insights from an immuno-dermatological perspective. Vaccines 9(9):944

3. Akinosoglou K, Tzivaki I, Marangos M (2021) Covid-19 vaccine and autoimmunity: awakening the sleeping dragon. Clin Immunol 226:108721

4. Cohen SR, Prussick L, Kahn JS et al (2021) Leukocytoclastic vasculitis flares following the COVID-19 vaccine. Int J Dermatol 60(8):1032-1033

5. Bostan E, Gulseren D, Gokoz O (2021) New-onset leukocytoclastic vasculitis after COVID-19 vaccine. Int J Dermatol 60(10):1305-1306

6. Kharkar V, Vishwanath T, Mahajan S, et al (2021) Asymmetrical cutaneous vasculitis following COVID-19 vaccination with unusual eosinophil preponderance. Clin Exp Dermatol https://doi.org/ 10.1111/ced.14797.

7. Erler A, Fiedler J, Koch A, et al (2021) A case of leukocytoclastic vasculitis after vaccination with a SARS-CoV2-vaccine - a case report. Arthritis Rheumatol https://doi.org/10.1002/art.41910

8. Mücke VT, Knop V, Mücke MM et al (2021) First description of immune complex vasculitis after COVID-19 vaccination with BNT162b2: a case report. BMC Infect Dis 21:958 
9. Liang I, Swaminathan S, Lee AYS (2021) Emergence of de novo cutaneous vasculitis post coronavirus disease (COVID-19) vaccination. Clin Rheumatol 1-2

10. Shakoor MT, Birkenbach MP, Lynch M (2021) ANCA-associated vasculitis following Pfizer-BioNTech COVID-19 vaccine. Am J Kidney Dis 78:611-613
Publisher's note Springer Nature remains neutral with regard to jurisdictional claims in published maps and institutional affiliations. 